\title{
Age factors in sex differences in open-field activity of rats
}

\author{
FRED P. VALLE and R. JEAN BOLS \\ The University of British Columbia, Vancouver, B.C., Canada V6T IWS
}

\begin{abstract}
Male and female Long-Evans rats were given open-field tests at 30 and/or 120 days of age. At 30 days of age, males and females did not differ in their open-field behavior. At 120 days of age, however, females locomoted more, reared more, and showed less wall-hugging than males, regardless of whether or not the animals had received tests in the open field at 30 days of age. The appearance of sex differences was due to an increase in the scores of the females between 30 and 120 days of age.
\end{abstract}

In both rats and hamsters, adult females typically engage in more open-field locomotor activity than adult males. Thirty-day-old hamsters, however, fail to show sex differences in open-field activity, and if such hamsters are retested in the open field as adults they fail to show the sex differences in locomotion that naive adults show (Swanson, 1969). Thus, in adult hamsters, the expression of sex differences in open-field activity depends upon the the developmental history of the animal with respect to the test environment.

The present study was undertaken to determine (a) if 30-day-old rats would show the sex differences in open-field activity characteristic of adults, and (b) if testing at $\mathbf{3 0}$ days of age would affect the expression of sex differences in open-field activity at 120 days of age. While several studies have shown that age and sex are each important determinants of rats' open-field behavior (e.g., Broadhurst, 1957; Furchgott, Wechkin and Dees, 1961; Hall, 1934; Valle, 1970, 1971), none apparently has examined the interaction between age and sex in this context. In addition to measuring amount of locomotion, the present study recorded several other aspects of rats' open-field behavior that have been shown to be sensitive to a variety of independent variables (Valle, 1970, 1971, 1972).

\section{METHOD}

\section{Subjects}

The subjects were 42 Long-Evans (black-hooded) derived rats bred from stock maintained in the psychology animal colony at the University of British Columbia.

\section{Apparatus \\ The open field was a square white board, $1.22 \mathrm{~m}$ on a side,}

This research was supported by the National Research Council of Canada, Grant APA-0266. Portions of the data were presented at the Canadian Psychological Association, June 1971. Requests for reprints should be sent to Fred P. Valle, Department of Psychology, University of British Columbia, Vancouver, B.C., Canada V6T IW5. divided by black lines into 16 equal squares. It rested on the floor on .05-m legs and had a $.3-\mathrm{m}$ wall around the periphery. A fine mesh screen covered the top of the field during testing. The field was located in a windowless room with illumination provided by a $150-\mathrm{W}$ white bulb suspended $.68 \mathrm{~m}$ above the floor of the field. The intensity of light was controlled by a transformer and was set at $.2 \mathrm{fc}$ as measured by a Trilux footcandle meter on the floor of the field with the mesh screen in place.

\section{Procedure}

The subjects were born in four litters. Prior to weaning, the rats received three brief handlings when the maternity cages were cleaned. Following weaning at 21 days, littermates were placed in a group cage and assigned to conditions randomly within litters. Ten males and 11 females were assigned to groups that received 3 open-field tests beginning at 30 days of age and an additional 10 open-field tests beginning at 120 days of age. These animals are hereafter designated as "experienced" subjects. Ten males and 11 females were assigned to groups that received only 10 open-field tests beginning at 120 days of age. These animals are hereafter designated as "naive" subjects. The tails of all rats were marked with colored ink for identification purposes.

Between 27 and 29 days of age, all subjects received daily handlings, 5 min in length. On Days 30-32, the experienced subjects received three 5-min open-field tests. On each test, the rat was carried individually to the testing room in a small cardboard box, tested, and then returned to a holding cage in the colony room, i.e., the subject was not returned to the group home cage until all subjects in that cage had been tested. Males within a litter were tested first, then the females. Order of testing over days remained constant. After each test, the field was washed with a mild vinegar-water solution. All tests were conducted between 1,700 and $2,000 \mathrm{~h}$.

Following the initial testing of the experienced subjects, all animals were left undisturbed, except for being placed in like-sex group cages at approximately 50 days, until 117 days when all subjects received 3 days of handling followed by 10 open-field tests as described above.

On all tests, the dependent variables were: (a) number and sequence of squares entered, with "entrance" defined as placing at least both forepaws in a square; (b) number of rears; (c) number of seconds spent in the four central (nonwalled) squares; (d) number of seconds spent grooming, and (e) number of boluses deposited.

Throughout the experiment, the rats were maintained on ad-lib food and water and under 24-h illumination. Over the course of the experiment, 5 males and 5 females died, and the final number of subjects in each condition was as follows: experienced males, 6; experienced females, 10; naive males, 9; naive females, 7. Thus, 32 animals completed the experiment. 


\section{RESULTS}

\section{Total Squares: 30-Day Tests}

It can be seen from Figure 1 that there was little difference in the number of squares entered by 30 -day-old males and females. Both sexes showed a decline in locomotion over the three tests. Analysis of variance showed a nonsignificant sex effect $(F<1)$, a significant tests effect $(F=12.79$, $\mathrm{df}=2 / 28, \mathrm{p}<.001)$, and a nonsignificant interaction $(F=1.28, \mathrm{df}=2 / 28)$.

\section{Total Squares: 120-Day Tests}

Figure 1 shows that at 120 days of age, females locomoted more than males, regardless of the testing history of the animal. Analysis of the data for the experienced animals showed a significant sex effect $(\mathrm{F}=23.47, \mathrm{df}=1 / 14, \mathrm{p}<.001)$, a nonsignificant test effect $(F<1)$, and a significant interaction $(\mathrm{F}=4.03$, df $=9 / 126, \mathrm{p}<.001)$. This interaction is apparently due to the concave nature of the male's curve over the first few tests compared with the relatively flat curve of the females.

Analysis of the results for the naive animals indicated a significant sex effect $(F=14.66, \mathrm{df}=1 / 14$, $\mathrm{p}<.01)$, a significant tests effect $(\mathrm{F}=9.66$, $\mathrm{df}=9 / 126, \mathrm{p}<.001$ ), and a nonsignificant interaction $(\mathrm{F}=1.49, \mathrm{df}=9 / 126)$. The significant tests effect reflects the decline in locomotion that occurred in both sexes over the first three or four tests.

\section{Rears: 30-Day Tests}

As shown in Figure 2, there was no difference in frequency of rearing between males and females at 30 days of age. For both sexes, rearing declined over tests. Analysis of variance indicated a nonsignificant sex effect $(F<1)$, a significant tests effect

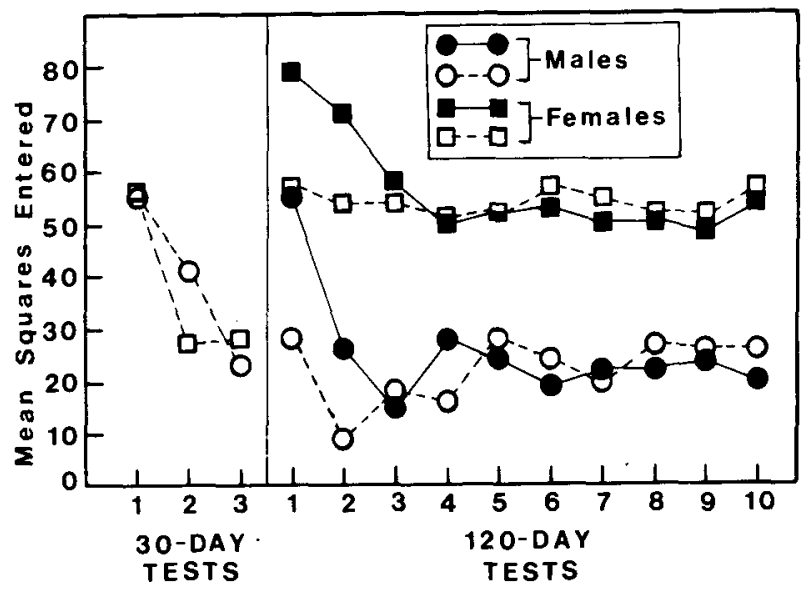

Figure 1. Mean number of squares entered during 5-min openfield tests by rats which received tests at both 30 and 120 days of age (open figures) and rats which received tests only at 120 days of age (filled figures).

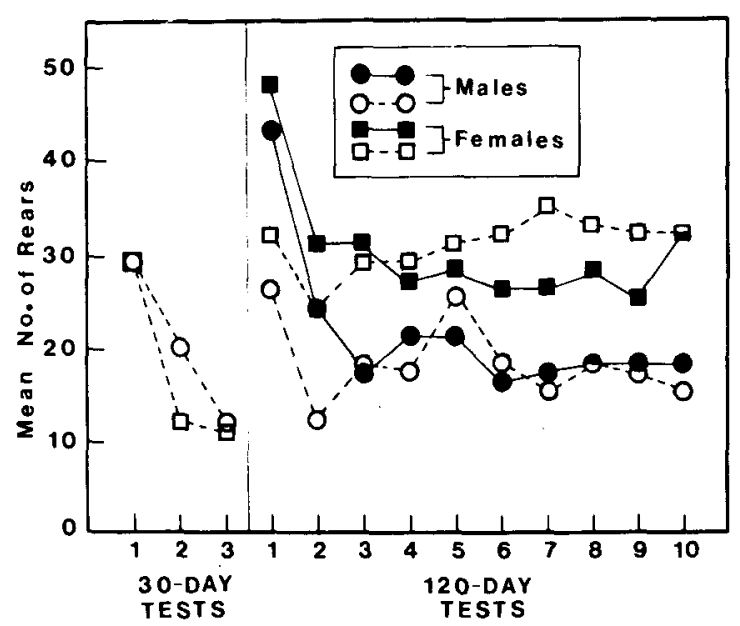

Figure 2. Mean number of rears during 5-min open-field tests by rats which received tests at both 30 and 120 days of age (open figures) and rats which received tests only at 120 days of age (filled figures).

$(\mathrm{F}=22.27, \mathrm{df}=2 / 28, \mathrm{p}<.001)$, and a nonsignificant interaction $(F=1.17, \mathrm{df}=2 / 28)$.

\section{Rears: 120-Day Tests}

It can be seen from Figure 2 that, at 120 days of age, both experienced and naive females reared more than their male counterparts. Analysis of the results for the experienced animals gave a significant sex effect $(\mathrm{F}=10.32$, $\mathrm{df}=1 / 14, \mathrm{p}<.01)$, a significant tests effect $(F=2.90, \mathrm{df}=9 / 126, \mathrm{p}<.05)$, and a nonsignificant interaction $(\mathrm{F}=1.44$, df $=9 / 126)$. The tests effect is due to an initial decline and subsequent recovery in rearing for both sexes.

Analysis of the data for the naive animals gave a significant sex effect $(F=6.99, \mathrm{df}=1 / 14, \mathrm{p}<.05)$, a significant tests effect $(F=19.71$, df $=9 / 126$, $\mathrm{p}<.001)$, and a nonsignificant interaction $(\mathrm{F}<1)$. It can be seen in Figure 2 that rearing declined over the initial three or four tests for both sexes.

\section{Thigmotactic Ratio: 30-Day Tests}

The thigmotactic ratio is a measure of the wallhugging or thigmotactic tendencies of the animal and is derived by dividing the number of central squares entered by the total number of squares entered. Hence, the bigger the ratio, the more prone was the subject to leave the peripheral squares and enter the central area of the field. As can be seen in Figure 3, at 30 days of age, males and females showed similar degrees of wall-hugging. Analysis of these data showed nonsignificant sex, tests, and interaction effects (all Fs $<1$ ).

\section{Thigmotactic Ratio: 120-Day Tests}

Figure 3 shows that adult females were more inclined to leave the peripheral walls than were adult males. Analysis of the results for experienced animals 


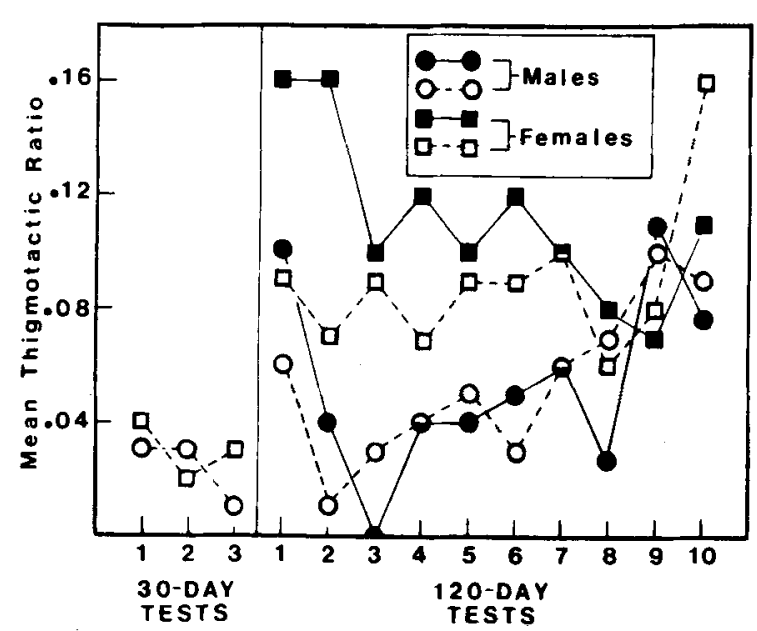

Figure 3. The ratio of central squares entered to total squares entered during 5 -min open-field tests by mis which received tests at both 30 and 120 days of age (open figures) and rats which received tests only at 120 days of age (filled figures).

indicated a significant sex effect $(F=4.80$, df $=$ $1 / 14, p<.05)$, a nonsignificant tests effect $(F=1.63$, df $=9 / 126)$, and a nonsignificant interaction $(F<1)$.

Analysis of the data for the naive animals showed a significant sex effect $(F=6.68, \mathrm{df}=1 / 14, \mathrm{p}<.05)$, a nonsignificant tests effect $(F=1.55, \mathrm{df}=9 / 126)$, and a nonsignificant interaction $(F=1.53$, $\mathrm{df}=$ 9/126).

\section{Seconds in Center}

The time scores yielded essentially the same picture as the thigmotactic ratios: nonsignificant sex effects at 30 days of age but significant sex effects at 120 days of age.

\section{Grooming Time}

There were no significant differences in time spent grooming at either 30 or 120 days of age.

\section{Number of Boluses}

There were no significant sex differences in frequency of defecation at either $\mathbf{3 0}$ or $\mathbf{1 2 0}$ days of age, although there was a marginally significant interaction $(F=3.06$, df $=2 / 28, .05<p<.10)$ between sex and tests at 30 days of age, with females showing no change in frequency of defecation over the three tests while males showed an increase in frequency of defecation over the three tests. No such interactions appeared at 120 days of age.

\section{Post Hoc Analyses}

Inspection of Figures 1, 2, and 3 suggests the following conclusions. (1) Males and females do not differ on these measures at 30 days of age. (2) Naive males at 120 days of age do not differ from males at 30 days of age. (3) Naive females at 120 days of age do differ from females at 30 days of age.
The first of these conclusions is supported by the analyses presented above. To determine the plausibility of the remaining two hypotheses, a mean score for each animal for its first three tests (at $\mathbf{3 0}$ days of age for the experienced subjects and at 120 days of age for the naive subjects) was obtained and $F$ tests comparing (i) 30- vs. 120-day-old males and (ii) 30vs. 120-day-old females were computed. These tests are orthogonal to the earlier analyses.

\section{Number of Squares Entered}

There was no significant difference between 30 and 120-day-old males (means, 39.6 and 32.3, respectively) in number of squares entered $(F<1)$. There was, however, a significant difference between 30- and 120-day-old females (means, 36.8 and 69.4, respectively) on this measure $(F=13.50, \mathrm{df}=1 / 15$, $\mathrm{p}<.005)$.

\section{Number of Rears}

There was no significant differences between 30 and 120-day-old males (means, 20.3 and 27.9, respectively) in frequency of rearing $(F=3.16$, $\mathrm{df}=1 / 13)$. There was, however, a significant difference between 30- and 120-day-old females (means 17.3 and 36.6 , respectively) in frequency of rearing $(F=28.61, \mathrm{df}=1 / 15, \mathrm{p}<.001)$.

\section{Thigmotactic Ratios}

There was no significant difference between 30 and 120-day-old males (means, .02 and .04 , respectively) in thigmotactic ratios $(\mathrm{F}=2.35, \mathrm{df}=1 / 13)$. There was, however, a significant difference between 30 - and 120-day-old females (means, .03 and .14 , respectively) in thigmotactic ratios $(F=27.53$, df $=1 / 15, \mathrm{p}<.001$ ).

\section{DISCUSSION}

The results of this study show that for every activity measure on which 120-day-old males and females differed significantly-number of squares entered, number of rears, and degree of wall-hugging (thigmotactic ratios)-30-day-old rats failed to show significant differences due to sex. The results were equally conclusive in showing that those subjects which failed to differ at $\mathbf{3 0}$ days of age, exhibited typical sex differences in open-field activity at 120 days of age. Hence, unlike the case with hamsters (Swanson, 1969), the expression of sex differences in the open-field activity of rats appears to be independent of previous experience in the test situation.

For those measures that yielded adult sex differences, it is clear in each case that the difference was due to an increase in the females' scores between 30 and 120 days of age. Thus, the appearance of sex differences in activity is due to some change that occurs in the females between 30 and 120 days of age. 
An immediate candidate for such a change would be the onset of ovarian activity and the consequent change in the female's hormonal state. This hypothesis is consistent with the observation that, in adult female rats, amount of open-field activity is greater at estrus than at diestrus (Birke \& Archer, 1975; Quadagno, Shryne, Anderson, \& Gorski, 1972). It should also be noted in this regard that when rats are maintained under $24-\mathrm{h}$ illumination, as in the present study, the females, once mature, typically enter a state of continual estrus (Wurtman, 1967). Persistent estrus, of course, would accentuate sex differences between groups of male and female rats if those differences were due, in some manner, to ovarian hormones.

It would be valuable to know (1) when sex differences in open-field activity first appear in rats, (2) whether their appearance is correlated with the onset of estrous cycles, and (3) whether their appearance can be accelerated by injections of estrogen and progesterone.

It would also be valuable to have information on other strains of rats to determine the generality of the effects found in the present study. In this regard, it is, perhaps, encouraging that Valle (1970) found no significant interactions between sex and strain on several measures of open-field activity using the Long-Evans strain and a strain of agouti-colored, nonhooded rats.

\section{REFERENCES}

Birke, L. I. A., \& Archer, J. Open-field behaviour of oestrous and dioestrous rats: Evidence against an 'emotionality' interpretation. Animal Behaviour, 1975, 23, 509-512.

Broadhurst, P. L. Determinants of emotionality in the rat. $I$. Situational factors. British Journal of Psychology, 1957, 48, 1-12.

Furchtgott, E., Wechicin, S., \& DeEs, J. W. Open-field exploration as a function of age. Journal of Comparative and Physiological Psychology, 1961, 54, 386-388.

HaLL, C. S. Drive and emotionality: Factors associated with adjustment in the rat. Journal of Comparative Psychology, 1934, 17, 89-108.

Quadagno, D. M., Shryne, J., Anderson, C., \& Gorski, R. A Influence of gonadal hormones on social, sexual, emergence, and open field behaviour in the rat (Rattus norvegicus). Animal Behaviour, 1972, 20, 732-740.

Swanson, H. I. Interaction of experience with adrenal and sex hormones on the behaviour of hamsters in the open field test. Animal Behaviour, 1969, 17, 148-154.

VALLE, F. P. Effects of strain, sex, and illumination on open-field behavior of rats. American Journal of Psychology, 1970, 83, 103-111.

VALLE, F. P. Rats' performance on repeated tests in the open field as a function of age. Psychonomic Science, 1971, 23, 333-335.

VALLE, F. P. Free and forced exploration in rats as a function of between- vs with-Ss design. Psychonomic Science, 1972, 29, 11-13.

Wurtman, R. J. Effects of light and visual stimuli on endocrine function. In L. Martini \& W. F. Ganong (Eds.), Neuroendocrinology (Vol. II). New York: Academic Press, 1967. Pp. 19-59.

(Received for publication December 8, 1974; revision accepted August 2, 1976.) 